\title{
Top-Down Tandem Mass Spectrometry of tRNA Via Ion Trap Collision-Induced Dissociation
}

\author{
Teng-Yi Huang, Jian Liu, and Scott A. McLuckey \\ Department of Chemistry, Purdue University, West Lafayette, Indiana, USA
}

Transfer RNA is a class of highly modified and structured non-coding RNA molecules generally comprised of 74-95 nucleotides. In this study, tandem mass spectrometry of intact multiply charged tRNA anions of roughly $25 \mathrm{kDa}$ in mass has been demonstrated using a quadrupole/time-of-flight tandem mass spectrometer adapted for ion/ion reaction studies. The sample proved to be a mixture of tRNA molecules. The mass of the most abundant component of the mixture was not consistent with that of the nominal identity of the tRNA from the supplier, viz., tRNA $^{\text {Phe, }}$; rather, the mass was consistent with tRNA ${ }^{\text {Phe }}$ bearing an incomplete $3^{\prime}$-terminus. Multiply-charged anions from the major components were isolated in the gas phase and subjected to ion trap collision-induced dissociation without subsequent ion/ion reactions. Abundant fragments from the $5^{\prime}$ - and $3^{\prime}$-termini of the molecule could be used to identify the major component as tRNA ${ }^{\text {Phe }}-3^{\prime}$ adenosine (without $3^{\prime}$-phosphorylation). Roughly $15 \%$ of the primary sequence of the intact tRNA was unambiguously reflected in the product ion spectrum. The existence of a possible tRNA ${ }^{\text {Phe }}$ variant and the intact $\mathrm{tRNA}^{\mathrm{Phe}}$ was also supported by ion trap CID data. The multiply-charged fragment ions derived from tRNA ${ }^{\text {Phe }}$-3'adenosine were further charge-reduced to mostly singly- and doubly-charged species via proton transfer ion/ion reactions with benzoquinoline cations. The resulting reduction in spectral overlap and charge state ambiguity simplified interpretation of the product ion spectrum and allowed for the identification of product ions from roughly $60 \%$ of the sequence. (J Am Soc Mass Spectrom 2010, 21, 890-898) (c) 2010 American Society for Mass Spectrometry

$\mathrm{T}$ Iransfer ribonucleic acids (tRNAs), which assume their activities after proper maturation from pretRNAs, play important roles in cellular function. In prokaryotic cells, precursor transcripts are processed through 5 ' - and 3 '-terminal cleavage and posttranscriptional modifications. In eukaryotic cells, the complete removal of introns by splicing is also involved in the process of tRNA maturation [1]. In addition, there are over 100 known modified nucleosides that occur in RNAs, and most of them are found in tRNAs [2,3]. The modifications are functionally important to tRNAs [4]. They can affect not only the structure and stability of tRNA, but also the maturation process. Modifications in the anticodon region play important roles in translation and cell growth. Although most of the modifications outside the anticodon region to date have not been noted to have an effect on phenotype, some of them have been found to have critical impact on maturation and stability of tRNAs [5]. In addition, editing at the 5'and $3^{\prime}$-termini is also important in protein biosynthesis. For example, the $3^{\prime}$-terminal trinucleotide sequence cytidine-cytidine-adenosine (CCA), which is encoded in the prokaryote gene but is added after $3^{\prime}$-terminal cleavage in eukaryotic cells, is a main interaction site for

Address reprint requests to Dr. S. A. McLuckey, Department of Chemistry, Purdue University, 560 Oval Drive, West Lafayette, IN 47907-2084, USA. E-mail: magnus.palmblad@angstrom.uu.se
23S rRNA, and the attachment site for activated amino acids [6]. Clinically, diverse human mitochondrial diseases have been linked to mutations on mitochondrial tRNA genes or the modification of mitochondrial tRNAs [7, 8].

The conventional biochemical tools for analysis of RNA species, such as Sanger sequencing of cDNA, are time-consuming and often ineffective for characterizing modified RNA [9]. Several mass spectrometry-based methods have been developed for RNA sequencing, such as mass analysis of sequencing ladders generated via $5^{\prime} \rightarrow 3^{\prime}$ and $3^{\prime} \rightarrow 5^{\prime}$ exonucleases, or a combination of various endonuclease digestions, or the reconstruction of sequences based on tandem mass spectrometrygenerated characteristic fragment ions. These mass spectrometry-based techniques offer rapid and sensitive alternatives for the characterization of RNA species [10].

Analogous to "bottom-up" approaches in proteomics research, analysis of chemically cleaved or enzymatically digested tRNA species by tandem mass spectrometry has successfully determined primary structures and modified bases [11-14]. However, loss of the intact tRNA mass information, congestion of peaks in a narrow mass range, and discrimination of certain oligomers from ionization of complex mixtures can limit the analytical utility of digestion-based approaches. In addition, due to their structural similarity, purification
(C) 2010 American Society for Mass Spectrometry. Published by Elsevier Inc. $1044-0305 / 10 / \$ 32.00$

doi:10.1016/j.jasms.2009.12.007
Published online December 28, 2009

Received November 5, 2009

Revised December 15, 2009

Accepted December 16, 2009 
of individual tRNA molecules is difficult [15]. Enzymatic digestions of a mixture of tRNAs can lead to an even more complex mixture of precursor ions and can add further ambiguity regarding determination of the identities and structures of the mixture components. Therefore, separation of digested tRNA oligomers by liquid chromatography is usually needed before mass spectrometry analysis.

In contrast to the "bottom-up" approach, a "topdown" approach, which does not require enzymatic digestion of analytes before mass spectrometry analysis, has been demonstrated and applied in proteomics research [16-19]. Application of tandem mass spectrometry to intact protein ions, rather than to peptide ions, is very attractive in that the mass of the intact molecule can be directly associated with the structural information obtained via tandem mass spectrometry. This link, which is particularly important when the goal is to identify and characterize modified species, is lost in a digestion-based approach. However, elimination of the digestion step can introduce new challenges in the separation, ionization, and tandem mass spectrometry steps because they are conducted on whole proteins, rather than peptides. Nevertheless, the potential advantages of top-down approaches, particularly with respect to modified biopolymers, have motivated their continued development.

A key element in all top-down approaches is the derivation of structural information by dissociation of analyte ions in gas phase. DNA-derived anions as large as 108 residues long have been subjected to tandem mass spectrometry, with complete sequence information resulting from a DNA 50-mer [20]. However, the tandem mass spectrometry of ions derived from noncoding RNA oligomers of roughly 20 residues or more is relatively little explored. Less data are available on the dissociation patterns of oligoribonucleotide ions than for oligodeoxyribonucleotide ions [21]. Most RNA fragmentation studies have been focused on MALDIgenerated singly protonated RNA cations $[22,23]$ or on ESI-generated anions of short RNAs [24, 25] under beam-type collisional activation conditions. Anions derived from RNA show two main competing backbone cleavage pathways that give rise to complementary $\mathrm{c} / \mathrm{y}$-ions and $(\mathrm{a}-\mathrm{B}) / \mathrm{w}$-ions, respectively, whereas DNA anions are dominated by the cleavage pathway that gives rise to the latter type of complementary pair. Using ion trap collisional activation, it has been demonstrated for model 8-mer RNA anions that conditions can be established that strongly favor the $\mathrm{c} / \mathrm{y}$-ion formation pathways [26]. Specifically, precursor anions of low to moderate charge states and relatively low ion activation amplitudes favors $\mathrm{c} / \mathrm{y}$-ion formation such that relatively high levels of sequence information can be obtained while avoiding additional complexity associated with contributions from competing dissociation pathways and sequential fragmentation. Similar findings were subsequently made in the ion trap collisional activation of anions derived from the sense and anti- sense strands of a siRNA duplex [27]. Under FT-MS conditions, dissociation of RNAs modified covalently by structural probes has been reported using SORI-CID [28]. Despite the facile loss of the modified base, sufficient sequence information was obtained to localize the covalent modification. In addition, dissociation of noncovalently bound RNA/drug complexes has also been investigated using IRMPD [29] and SORI-CID [30]. In these studies, RNA of about $20 \sim 30$ nucleotides were used as model systems to mimic certain stem-loop structures of important RNA species of larger sizes. Extensive sequence coverage was generally obtained; however, sequence coverage gaps were usually observed for the RNA/chemical complexes, which indicate the regions of interactions between RNA and drugs. It is worth noting that the effects of hydrogen bonding on the dissociation behavior of DNA duplexes have been reported [31, 32]. Both the dissociation of hydrogen bonds and various covalent bond dissociation channels were observed. For each strand of siRNA, the secondary structure is usually minimal; therefore, no structure effects on gas-phase dissociation were observed. However, even for RNA stem-loops, no hydrogen-bonding effects on the dissociation behavior have been reported. In this study, previous findings were used to extend the "top-down" approach to a tRNA mixture using a commercial quadrupole/time-offlight tandem mass spectrometer adapted for ion/ion reaction studies [33]. This represents a particularly stringent test for top-down tandem mass spectrometry of nucleic acid anions due both to the size of the molecule and the high degree of intramolecular interactions that lead to the folding of tRNA into its canonical cloverleaf secondary structure and the functional L-shape tertiary structure.

\section{Experimental}

\section{Materials}

Methanol and glacial acetic acid were purchased from Mallinckrodt (Phillipsburg, NJ, USA). The yeast phenylalanine transfer RNA, piperidine, imidazole, benzoquinoline, and Amberlite IRN77 hydrogen form were obtained from Sigma-Aldrich (St. Louis, MO, USA).

RNA samples were desalted before mass spectrometry analysis. Desalting was carried out through the use of a cation exchange resin. Desalting of RNA [34] by the cation exchange resin (Amberlite IRN77 hydrogen form) was carried out as described below. The cation exchange resin was rinsed with DEPC- $\mathrm{H}_{2} \mathrm{O}$ and then mixed with $100 \mu \mathrm{L}$ tRNA stock solution of about 200 $\mu \mathrm{M}$ at room temperature for $10 \mathrm{~min}$ to reduce the cation adducts $\left(\mathrm{Na}^{+}\right.$or $\left.\mathrm{K}^{+}\right)$. RNA solutions for negative nanoelectrospray were prepared by diluting the desalted aqueous stock solutions to ca. $20-40 \mu \mathrm{M}$ in $20 / 80$ (vol/vol) isopropanol/water with addition of $25 \mathrm{mM}$ piperidine and $25 \mathrm{mM}$ imidazole [35]. Under optimal 
conditions, less than 1 nmol tRNA sample was typically used during data acquisition.

\section{Apparatus and Procedures}

All experiments were performed using a prototype version of QqTOF tandem mass spectrometer modified to allow for ion/ion reaction studies [33]. A home-built alternate atmospheric pressure chemical ionization (APCI) and nano-electrospray (ESI) source was coupled directly to the QqTOF instrument [36]. For protontransfer ion/ion reactions, ionization was accomplished via a nano-ESI emitter for the formation of $[\mathrm{M}-\mathrm{nH}]^{\mathrm{n}-}$ anions of tRNA, and an APCI source for the formation of singly protonated reagent cations. In brief, the tRNA samples were loaded into nano-electrospray emitters pulled from borosilicate capillaries (1.5 mm o.d., 0.86 $\mathrm{mm}$ i.d.) using a P-87 Flaming/Brown micropipet puller (Sutter Instruments, Novato, CA, USA). A stainless steel wire was inserted into the back of the capillary, and a potential of $0.8-1.1 \mathrm{kV}$ was applied to the wire for ionization. To perform ion trap CID in Q2, Q1 selected precursor ions were injected into Q2 at relatively low kinetic energy, and a dipolar rf signal with frequency in resonance with the precursor ions was then applied to induce ion trap CID. To facilitate the interpretation of the product ion spectra from top-down analysis of intact $t R N A$, proton transfer reactions using protonated benzoquinoline $\left(\mathrm{BQH}^{+}\right)$as reagent cations were employed to reduce the charge states of fragment ions. However, due to adduct formation, low-energy beam-type CID was performed in some cases, as described in the text, to remove the attached reagents. A typical scan function consisted of RNA anion injection, isolation of ions of interest by Q1 in RF/DC mode, ion trap CID in Q2, cation injection to Q2, mutual cation/ anion storage, and then mass analysis by TOF.

\section{Data Analysis}

The 51 yeast tRNA sequences were extracted from a much larger tRNA sequence database (http://www. trna.uni-bayreuth.de/) [37]. The masses of these sequences were then determined for matching with experimental data. The oligoribonucleotide molecular weight and electrospray series were calculated by using Mongo Oligo Mass Calculator (http:/ /library.med.utah. edu/masspec/mongo.htm) [38]. The theoretical CID fragment ions of intact tRNA anions were calculated manually. The average masses of the major components were determined from the nano-ESI spectrum using a deconvolution function, Bayesian Protein Reconstruct, from the BioAnalyst software (Applied Biosystems, Foster City, CA, USA). The post-ion/ion CID spectra were also processed by the centroid function of the BioAnalyst software to improve the signal to noise ratio.

\section{Results and Discussion}

An overall approach for top-down interrogation of ions from a tRNA mixture is summarized schematically in Figure 1. Before tandem mass spectrometry, the mixture is subjected to negative nanoelectrospray ionization followed by time-of-flight mass analysis. Molecular masses of individual tRNA components can be correlated with the theoretical molecular masses calculated from the published tRNA database [37]. Precursor ions of interest can then be selected via a mass filter and accumulated in the linear ion trap of the quadrupole/ time-of-flight instrument. Ion trap collision-induced dissociation of the multiply-charged tRNA anion leads to a mixture of products of various masses and charges. Singly-charged cations are introduced into the ion trap to reduce the charges of the product ions largely to

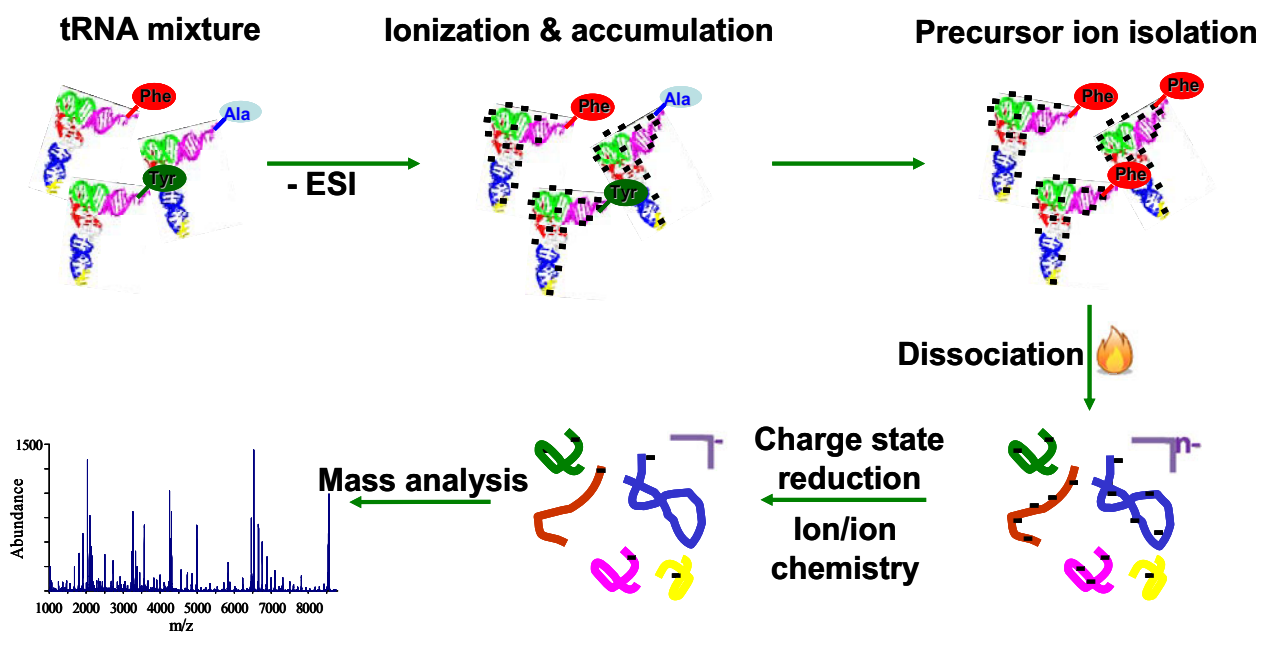

Figure 1. Schematic of the top-down tandem mass spectrometry of tRNA mixture components with ion/ion proton transfer reactions to facilitate spectral interpretation."The tRNA structure was reproduced with the kind permission of N. R. Voss." 
singly-charged anions. The resulting post-ion/ion reaction product ion spectrum is then interpreted with the aid of predicted product ion masses calculated from the tRNA with a matching mass from the database.

\section{MS and MS/MS of the Simple tRNA Mixture}

A well-known complication associated with ionization of nucleic acids is the possibility for the presence of mixtures of counter-ions, principally protons and metal ions, associated with phosphodiester linkages [34, 35]. This phenomenon compromises both mass determination and sensitivity and tends to become more problematic as the number of phosphodiester linkages increase (i.e., as the oligomer size increases). To determine molecular mass, a yeast tRNA ${ }^{\text {Phe }}$ sample of higher than $90 \%$ purity, as determined by the supplier based on activity, was subjected to cation exchange resin treatment before mass analysis in the negative ion mode. As shown in the supplementary data (Figure S-1A, which can be found in the electronic version of this article), the negative ion nano-ESI spectrum was not resolvable due to the high degree of adduction of counter ions other than protons before desalting. After $10 \mathrm{~min}$ of cation exchange resin treatment (Figure S-1B), five major components with multiple sodium adducts were distinguishable in each charge state in the nano-ESI spectrum. However, as shown in Figure S-1C, the relative abundances of the five components showed some variability after a prolonged cation exchange resin treatment (e.g., $30 \mathrm{~min}$ ), presumably due to differential absorption to the resin. Given the similarity in masses and the common procedure for isolation, likely all of the major components are tRNAs. Compared to conventional desalting methods, such as repeated ethanol precipitation, use of the cation exchange resin is time-efficient and can be used as a supplemental desalting method to remove residual salts in the biological RNA samples.
The average masses of the major components were determined from the nano-ESI spectrum using a deconvolution program from the BioAnalyst software. The mass values, which are assumed to contain no sodium adducts, were compared with the theoretical average masses calculated from the published yeast (Saccharomyces cerevisiae) tRNA database. The species giving rise to the third most abundant ion signal matches the predicted mass for yeast $\mathrm{tRNA}^{\text {Phe }}$ (ID RF7630) in the tRNA sequence database [37]. It has been shown that "purified" yeast tRNA ${ }^{\text {Phe }}$ usually contains more tRNA ${ }^{\text {Phe }}-3^{\prime}$ A (loss of the $3^{\prime}$-adenosine, including the $3^{\prime}$ phosphate, from tRNA $\left.{ }^{\text {Phe }}\right)$ than intact tRNA ${ }^{\text {Phe }}[39,40]$. Possibly the third most abundant component (measured $\mathrm{M}_{\mathrm{r}}=24,952.5 \pm 1.1$ ) corresponds to yeast tRNA $^{\text {Phe }}$ (calculated $\mathrm{M}_{\mathrm{r}}=24,951.3$ ), and the most abundant component (measured $\mathrm{M}_{\mathrm{r}}=24,622.0 \pm 0.4$ Da) corresponds to tRNA ${ }^{\text {Phe }}-3^{\prime}$ A without 3'-phosphorylation (calculated $\mathrm{M}_{\mathrm{r}}=24,622.1$ ). The second most abundant component, corresponding to a molecular mass of $24,264.1 \pm 1.5$, however, does not match any of the theoretical masses calculated, nor do those of the two relatively low abundance components.

The presence of intact $\operatorname{tRNA}^{\text {Phe }}$ in this sample is supported based on mass measurement. In general, however, the specific identities of tRNA species can be difficult to establish confidently based on molecular mass alone, in part because not all of the modifications have been identified. In addition, various posttranscriptional processes during tRNA maturation can change the molecular weight of tRNAs. Hence, it is desirable to derive sequence information and to localize modifications of various types, such as splicing, nucleoside modification, and editing on the $3^{\prime}-$ and $5^{\prime}-$ termini. To explore the extent of sequence information that can be derived from ion trap CID of a multiplycharged tRNA anion, the most abundant component of the ion mixture was first subjected to top-down tandem

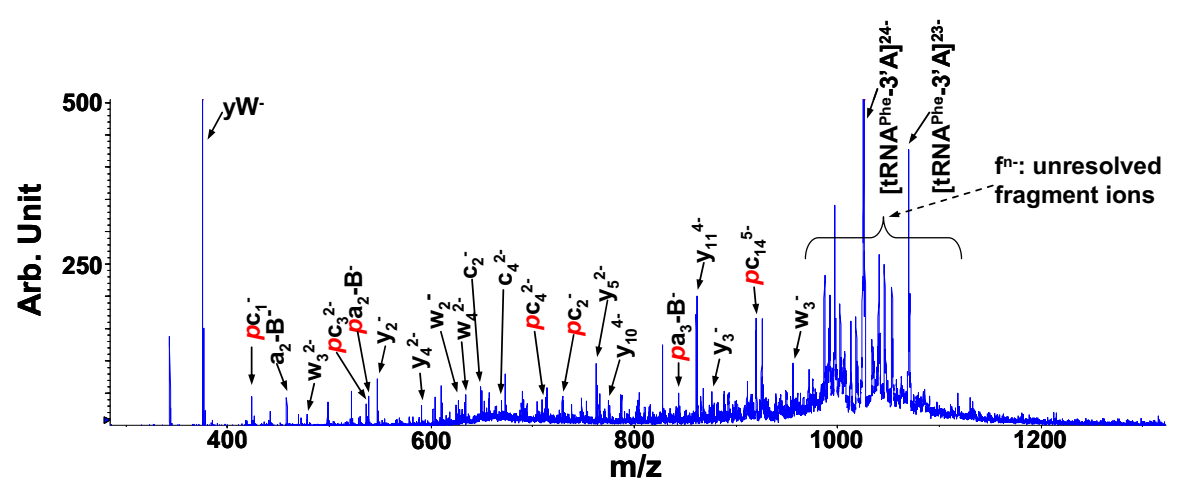

Figure 2. Ion trap CID of the $\left[\mathrm{tRNA}^{\mathrm{Phe}}-3^{\prime} \mathrm{A}\right]^{24-}$. High charge state fragment ions $\left(\mathrm{f}^{\mathrm{n}-}\right)$ tend to concentrate in a narrow region, and cannot be resolved. However, low charge state fragment ions, corresponding to the $5^{\prime}$ - and $3^{\prime}$-terminal ions, provide limited sequence coverage. The base loss of wybutosine is indicated as $\mathrm{yW}^{-}$. The prefix " $p^{\prime \prime}$ (red) indicates the retention of the $5^{\prime}$-phosphate on some of the $\mathrm{c}$ and a-B ions from the $5^{\prime}$-terminus. The $\left[\mathrm{tRNA}^{\mathrm{Phe}}-3^{\prime} \mathrm{A}\right]^{23-}$ ions were also observed due to the ion/molecule reaction between the isolated precursor ions [ $\left.\operatorname{tRNA}^{\mathrm{Phe}}-3^{\prime} \mathrm{A}\right]^{24-}$ and $n$ butylbenzenesulfonamide in the nitrogen gas tubing. 
mass spectrometry. The product ion spectrum derived from ion trap CID of the $[\mathrm{M}-24 \mathrm{H}]^{24-}$ species, the mass measurement for which is consistent with $\mathrm{M}$ being $\operatorname{tRNA}^{\mathrm{Phe}}-3^{\prime} \mathrm{A}$, is shown in Figure 2. The product ion spectrum is highly congested, particularly near the mass-to-charge value of the precursor ion. The resolving power of the TOF $(\mathrm{M} / \Delta \mathrm{M}$ FWHM $\sim 8000)$ is insufficient to determine the charge states of the high mass fragments even in the absence of peak overlap. The high degree of spectral complexity associated with the high mass and relatively high charge state fragment ions $\left(\mathrm{f}^{\mathrm{n}-}\right)$, which show extensive overlap, complicates the interpretation of the spectrum even further. However, relatively abundant fragments of low charge states that appear above the background can be assigned. These lower $\mathrm{m} / \mathrm{z}$ fragment ions, $p \mathrm{c} / \mathrm{c} / \mathrm{y}$ and $p(\mathrm{a}-\mathrm{B}) / \mathrm{a}-$ $\mathrm{B} / \mathrm{w}$ ions, correspond to the $5^{\prime}$ - and $3^{\prime}$-terminal ions of tRNA ${ }^{\text {Phe }}-3^{\prime} A$, but not to any other sequences in the published yeast tRNA database. Hence, sufficient information can be gleaned from the combination of mass measurement and the CID spectrum to further support the conclusion that $t R N A^{\text {Phe }}-3^{\prime} \mathrm{A}$ is the most abundant component in the partially purified tRNA sample.

To identify the other tRNAs in the sample, the [M $23 \mathrm{H}]^{23-}$ precursor ions of three of the most abundant tRNA components were subjected to top-down tandem mass spectrometry (supplementary Figure S-2). Due to the limited resolving power and the high spectral congestion, the interpretation of the spectra are limited to the $3^{\prime}$ - and $5^{\prime}$-terminal fragment ions at the lower $\mathrm{m} / \mathrm{z}$ range. In comparing the ion trap CID data, $\mathrm{tRNA}^{\text {Phe }}$ $3^{\prime} \mathrm{A}$ and the unknown component share similar fragmentation patterns (S-2A and S-2B). The base loss ion $(\mathrm{yW})^{-}$, two of the most abundant fragment ions, $\left(\mathrm{y}_{11}\right)^{4-}$ and $\left(p c_{14}\right)^{5-}$, and several low abundant ions corresponding to the $5^{\prime}$ - and $3^{\prime}$-termini of $\mathrm{tRNA}^{\text {Phe }}-3^{\prime} \mathrm{A}$ sequence were also observed in the CID spectrum of the unknown tRNA. Possibly, the unknown tRNA component is another tRNA ${ }^{\text {Phe }}$ variant with unclassified modifications or sequence variation between nucleotide positions $12 \sim 64$.

The identity of the third most abundant component as intact $t R N A^{\text {Phe }}$ is also supported by ion trap CID of the $[\mathrm{M}-23 \mathrm{H}]^{23-}$ precursor ions. In comparison to dissociation of the other two major components, $\operatorname{tRNA}^{\text {Phe }}-3^{\prime} \mathrm{A}$ and the unknown tRNA, sequence specific ions assigned to the $5^{\prime}$-terminus of intact $t R N A^{\text {Phe }}$ are similar to its loss of $3^{\prime}$-adenosine $\mathrm{tRNA}^{\text {Phe }}$ variants, and the ions assigned to the $3^{\prime}$-terminus of the intact tRNA $^{\text {Phe }}$ are $329.2 \mathrm{Da}$ higher in mass. As shown in Figure S-2C, several $p c$-ions (i.e., $p c_{3}, p c_{7}, p c_{8}$, and $p c_{14}$ ) corresponding to the $5^{\prime}$-terminus of the tRNA ${ }^{\text {Phe }}$ sequence were observed, while two y ions, $\left(\mathrm{y}_{12}\right)^{4-}$ and $\left(y_{5}\right)^{2-}$, corresponding to the $3^{\prime}$-terminus of the intact $\mathrm{tRNA}^{\text {Phe }}$ sequence rather than the $3^{\prime}$-truncated variants were observed. Although another $\mathrm{tRNA}^{\text {Phe }}$ sequence (ID RF7631, Mr = 24,966.3) with only two nucleotide differences (at nucleotide position 6 and position 67) has also been categorized in the tRNA sequence data- base [37], none of the major components are from tRNA $^{\text {Phe }}$ (ID RF7631) based on the mass and limited terminal fragment ions information obtained. The three major components are therefore identified as $\mathrm{tRNA}^{\text {Phe }}$ (ID RF7630) and its variants. It is noteworthy that the subtle variations in sequences would likely have been missed using a bottom-up approach, gel electrophoresis, and the conventional amino acid acceptor activity assay.

\section{Intact tRNA Sequencing Via Collision-Induced Dissociation and Proton Transfer Ion/Ion Reactions}

As demonstrated in Figure 2 and Figure S-2, one of the problems encountered in the dissociation of highly charged precursor ions is the wide charge distribution of fragment ions. The fragment ions of different masses and charges tend to concentrate in a narrow $\mathrm{m} / \mathrm{z}$ window that surrounds the $\mathrm{m} / \mathrm{z}$ of the precursor ion, thereby complicating the derivation of mass information when a high-resolution mass analyzer is not available. To access the sequence information present in the unresolved fragment ion population of Figure 2, proton transfer ion/ion reactions were applied to reduce the fragment ion charge states to mostly $1-$. Charge reduction of highly charged unmodified nucleic acid anions has been demonstrated by ion/ion reactions with protonated benzoquinoline cations in a 3-D ion trap [41] and a linear ion trap [27], respectively. Minimal adduct formation or fragmentation of parent ions was detected with this reagent. The simplification of the product ion spectrum via proton transfer ion/ion reactions allowed the assignment of product ions from the most favored dissociation channels by comparing the $m / z$ values to the expected singly charged fragment ions of $t \mathrm{RNA}^{\text {Phe }}$ $3^{\prime} \mathrm{A}$. However, due to relatively poor detector response for anions, due in part to the use of a relatively low accelerating voltage for the TOF (i.e., $4 \mathrm{kV}$ ), the postion/ion CID spectrum has a low signal-to-noise ratio, especially in the higher $m / z$ region. To distinguish signal from noise, the spectrum was processed using the centroid function provided by the BioAnalyst software, to convert peak distribution values into a single value by an intensity-weighted average algorithm to calculate the center of gravity of the peak. The centroid mass spectrum is shown in Figure 3, and the sequence coverage is summarized in Figure $4 \mathrm{a}$. The $p \mathrm{c} / \mathrm{y}$ and $p(\mathrm{a}-\mathrm{B}) / \mathrm{w}$ ion series are the major fragment ions, covering a wide range of sequence. The $\mathrm{c}$ and $\mathrm{a}-\mathrm{B}$ ion series, presumably resulting from the sequential $5^{\prime}$ phosphate loss and backbone cleavage (either order), were also observed. The unlabelled peaks in the spectrum correspond to fragment ions from other minor primary dissociation channels or from sequential fragmentation leading to products that contain neither the $5^{\prime}$ - nor $3^{\prime}$-terminus. Figure $4 \mathrm{~b}$ summarizes the sequence coverage on a secondary structure model, which shows that ion trap CID and proton transfer reaction reveal most of 


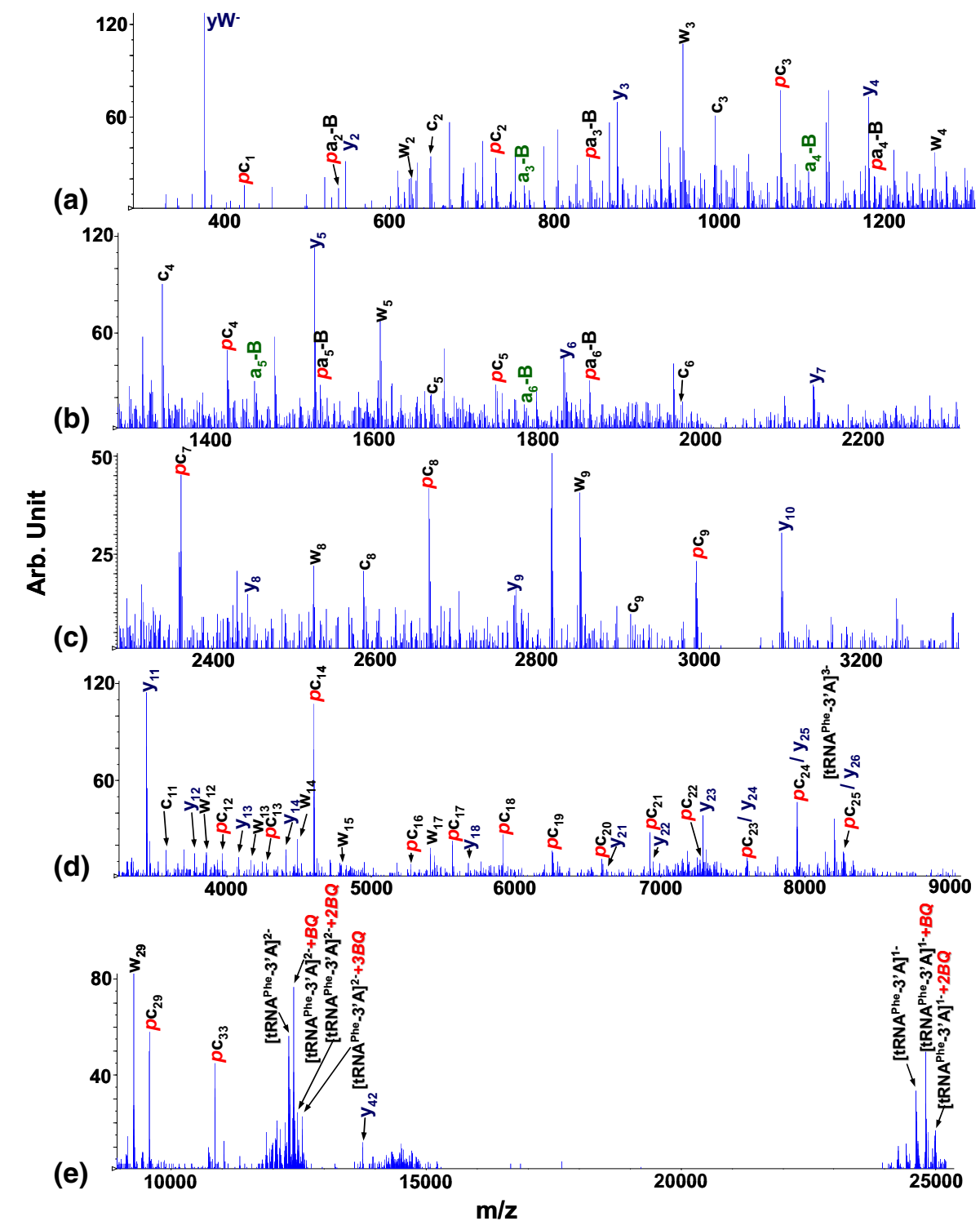

Figure 3. Post-ion/ion ion trap CID spectrum of $\left[\mathrm{tRNA}{ }^{\mathrm{Phe}}-3^{\prime} \mathrm{A}\right]^{24-}$. The base loss of wybutosine is indicated as $\mathrm{yW}^{-}$. $\mathrm{BQ}$ (red) indicates the attachment of benzoquinoline.

the sequence except the variable loop and part of the anticodon loop. In comparison to the pre-ion/ion CID data (Figure 2), in which fragments from less than 15\% of the sequence could be clearly identified, the combination of $5^{\prime} \mathrm{P}-\mathrm{O}$ bond or $3^{\prime} \mathrm{C}-\mathrm{O}$ bond cleavage in the post-ion/ion reaction data covered $60 \%$ of the tRNA ${ }^{\text {Phe }}$ $3^{\prime} \mathrm{A}$ sequence. Due to the lower abundances and less stable ion signals from the other components, no postion/ion CID spectra for the intact tRNA ${ }^{\text {Phe }}$ and the uncategorized variant have been obtained.

There are several contributing factors that currently limit the ability of the approach outlined here to fully characterize the primary tRNA structure, some of which are instrumental in nature while others are, perhaps, more fundamental in nature. For example, although the centroid algorithm can improve the quality of the spectra, the accurate mass assignment of peaks of low abundance is compromised, especially in the higher $\mathrm{m} / \mathrm{z}$ region where the isotopic distribution of the low abundant fragment ions is distorted or not complete due to poor ion statistics. Consequently, the mass difference of $1 \mathrm{Da}$ between $\mathrm{U}$ and $\mathrm{C}$ is not determined unambiguously at high mass range. The low signal levels, particularly at high $\mathrm{m} / \mathrm{z}$, are due in part to poor detection efficiency for high mass anions with the current apparatus. For the same reason, the nucleosides with the same nominal mass, such as $\mathrm{C}$ (cytidine) and $\mathrm{Cm}\left(2^{\prime}-\mathrm{O}\right.$-methylcytidine), $\mathrm{U}$ (uridine) and $\psi$ (pseudouridine), and $\mathrm{m}^{7} \mathrm{G}$ (7-methylguanosine) and $\mathrm{m}^{2} \mathrm{G}$ ( $N^{2}$-methylguanosine), cannot be distinguished, even when the mass measurement accuracy of the instrument is adequate when the ion statistics are good. The low signal levels at high $\mathrm{m} / \mathrm{z}$ may be ameliorated via the use of "ion parking" [42], a gas-phase concen- 

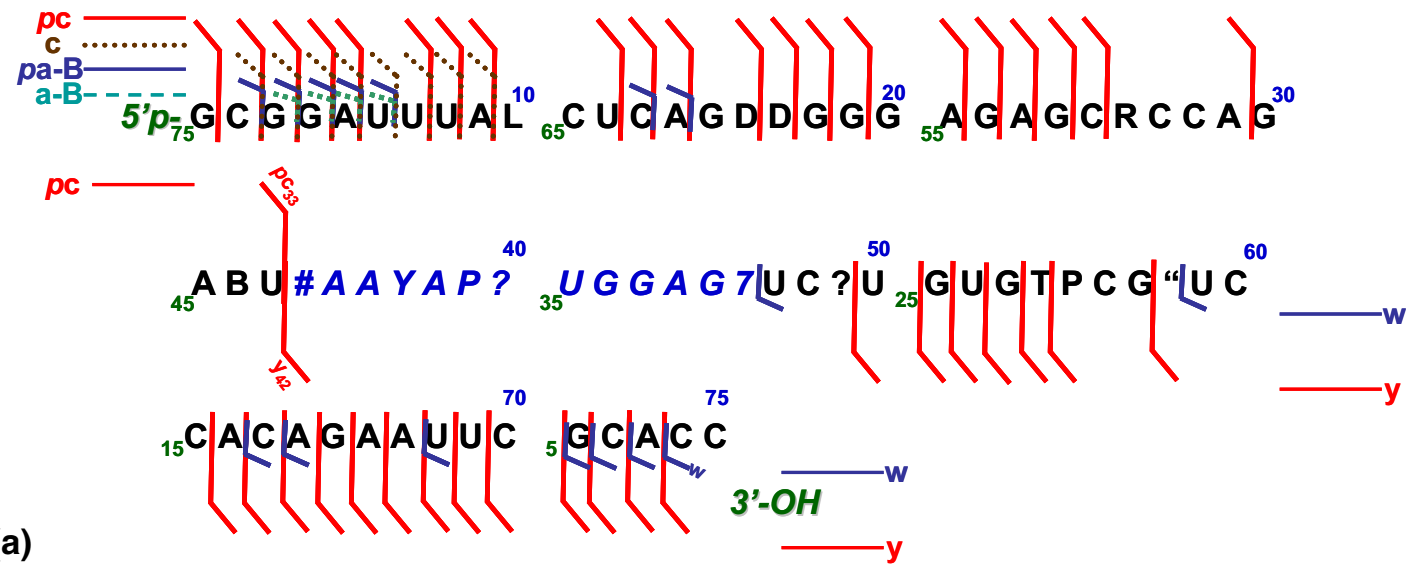

(b)

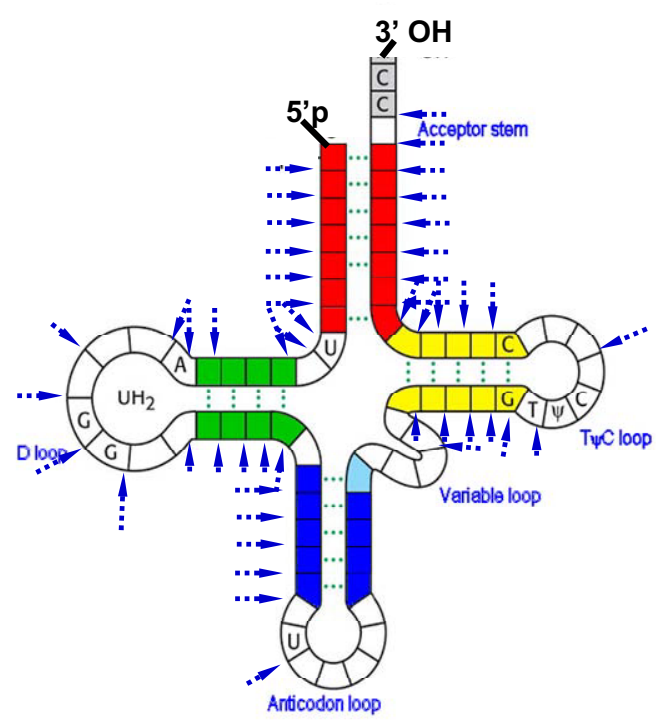

\begin{abstract}
Figure 4. Sequence coverage from post-ion/ion ion trap CID of the $\left[\mathrm{tRNA}^{\mathrm{Phe}}-3^{\prime} \mathrm{A}\right]^{24-}$. (a) Summary of backbone cleavages resulting from ion trap CID of the $\left[\mathrm{tRNA}^{\mathrm{Phe}}-3^{\prime} \mathrm{A}\right]^{24-}$ anion. Modified nucleosides: \# (Gm, 2'-O-methylguanosine), B (Cm, 2'-O-methylcytidine), "( $\mathrm{m}^{1} \mathrm{~A}, 1$-methyladenosine), ? $\left(\mathrm{m}^{5} \mathrm{C}, 5\right.$-methylcytidine ), 7 ( $\mathrm{m}^{7} \mathrm{G}, 7$-methylguanosine), $\mathrm{D}$ (D, dihydrouridine), $\mathrm{L}\left(\mathrm{m}^{2} \mathrm{G}, \mathrm{N}^{2}\right.$ methylguanosine), $\mathrm{P}\left(\psi\right.$, pseudouridine), $\mathrm{R}\left(\mathrm{m}_{2}^{2} \mathrm{G}, \mathrm{N}^{2}, \mathrm{~N}^{2}\right.$-dimethylguanosine), and $\mathrm{Y}$ (yW, wybutosine). (b) The backbone cleavages are indicated on a cloverleaf secondary structure of the tRNA ${ }^{\text {Phe }}-3^{\prime}$ A molecule by the arrows.
\end{abstract}

tration technique based on ion/ion chemistry that can be used to increase precursor ion signals for some charge states. This is not, however, a general solution for the poor detection efficiency. In any case, even with adequate signal levels, distinguishing isomeric nucleosides is problematic via tandem mass spectrometry. However, the most common modified nucleoside, pseudouridine, has been distinguished from its isomer, uridine, via nucleoside-specific chemical derivatization [43]. This method might be amenable for the top-down approach demonstrated here.

A fundamental issue that is not limited to the apparatus used in this work is that of the incomplete sequence coverage via CID. Previous work with the gas-phase dissociation of small single-stranded RNAs suggests that the dissociation channels that lead to complementary c/y-ions are less nucleobase dependent than the channels that lead to complementary $(\mathrm{a}-\mathrm{B}) / \mathrm{w}$ ions. The $\mathrm{c} / \mathrm{y}$-ion channels are favored at low excitation amplitudes and moderate-to-low charge states. Nevertheless, regions of the tRNA anions show no evidence for the formation of sequence informative products under any conditions explored in this study. Furthermore, the ion trap CID product ion spectrum of tRNA showed many relatively abundant nonspecific fragment ions that likely arise from sequential fragmentations. Much less sequential fragmentation was noted in the ion trap CID of siRNA single strand anions [27]. The behavior of the tRNA anions may well be accounted for by the high degree of intramolecular interactions associated with native tRNA, which can stabilize the ion and therefore require several bond cleavages before fragments separate from one another. It is not clear to what extent the condensed-phase structure of tRNA is 
preserved during the electrospray ionization process. However, the ready observation of the duplex structure of siRNA anions in this instrument [27] is evidence that much of the hydrogen bonding already present in tRNA in solution may survive into the gas phase. Hence, the lack of fragmentation from within the anticodon loop, for example, may reflect that this region is particularly stable to fragmentation due to intramolecular hydrogen bonding. It has been demonstrated that disruption of the protein gas-phase structure through various means allows for the gas-phase dissociation of large protein ions that are otherwise difficult to fragment by conventional means [44]. Perhaps analogous approaches might also be useful with tRNA.

\section{Conclusions}

In this study, a "top-down" mass spectrometry approach has been demonstrated for the major components of a tRNA mixture using a commercial quadrupole/ time-of-flight tandem mass spectrometer adapted for ion/ion reaction studies. In contrast to the "bottomup" approach, no enzymatic digestion, which precludes determination of the mass of the intact precursor, is required. Therefore, it was possible to observe that the sample was comprised of multiple components. Molecular masses of individual tRNA components were obtained based on the negative nano-ESI spectrum and were correlated with the theoretical molecular masses calculated from the published tRNA database. In addition, limited 5' - and 3'-structural information of the three major tRNA components was obtained from the low mass region of the ion trap CID product ion spectra. Based on the molecular mass and the low mass 5' - and $3^{\prime}$-fragment ions, the tRNAs can be identified by matching with the published tRNA database. To facilitate the interpretation of the fragment ion spectrum, the multiplycharged fragment ions of the most abundant species, tRNA $^{\text {Phe }}-3^{\prime} A$, were then further charge reduced to mostly singly and doubly charged species via proton transfer ion/ion reaction with benzoquinoline cations. The simplified post-ion/ion fragment ion spectrum provides improved sequence coverage, thereby enhancing the ability to characterize the molecule.

Recently, increasing attention has been focused on transcriptomics. Non-coding RNAs, previously thought to be irrelevant, are now thought to play important parts in signaling and regulatory events [45, 46]. Most of these non-coding RNAs are heavily processed from transcripts to become functional molecules through various processes, such as capping, splicing, polyadenylation, modification, editing, and $5^{\prime}-$ and/or $3^{\prime}$-end processing. These post-transcriptional processes affect the folding, protein interaction and subcellular localization of RNAs [47, 48]. The top-down approach demonstrated here, as illustrated in Figure 1, can be adapted to identify other non-coding RNAs and their modifications from a simple mixture. However, such a strategy stands to benefit from further developments in instrumentation, complementary dissociation approaches that can provide expanded sequence coverage, and more comprehensive databases to support the identification of modified RNAs.

\section{Acknowledgments}

The authors acknowledge support for this research by the National Science Foundation under CHE- 0808380 and by the Purdue Research Foundation.

\section{Appendix A Supplementary data}

Supplementary material associated with this article may be found in the online version at doi:10.1016/ j.jasms.2009.12.007.

\section{References}

1. Schafer, B. RNA Maturation in Mitochondria of S. cerevisiae and S. pombe. Gene 2005, 354, 80-85

2. Limbach, P. A.; Crain, P. F.; McCloskey, J. A. Summary: The Modified Nucleosides of RNA. Nucleic Acids Res 1994, 22, 2183-2196.

3. Agris, P. F. Decoding the Genome: A Modified View. Nucleic Acids Res. 2004, 32, 223-238.

4. Helm, M. Post-Transcriptional Nucleotide Modification and Alternative Folding of RNA. Nucleic Acids Res. 2006, 34, 721-733.

5. Alexandrov, A.; Chernyakov, I.; Gu, W.; Hiley, S. L.; Hughes, T. R. Grayhack, E. J.; Phizicky, E. M. Rapid tRNA Decay Can Result from Lack of Nonessential Modifications. Mol. Cells 2006, 21, 87-96.

6. Mörl, M.; Marchfelder, A. The Final Cut. The Importance of tRNA 3'-Processing. EMBO Rep. 2001, 2, 17-20.

7. Levinger, L.; Mörl, M.; Florentz, C. Mitochondrial tRNA 3' End Metabolism and Human Disease. Nucleic Acids Res. 2004, 32, 5430-5441.

8. Kirino, Y.; Suzuki, T. Human Mitochondrial Diseases Associated with tRNA Wobble Modification Deficiency. RNA Biol. 2005, 2, 41-44.

9. Hahn, C. S.; Strauss, E. G.; Strauss, J. H. Dideoxy Sequencing of RNA Using Reverse Transcriptase. Methods Enzymol. 1989, 180, 121-130.

10. Thomas, B.; Akoulitchev, A. V. Mass Spectrometry of RNA. Trends Biochem. Sci. 2006, 31, 173-181.

11. McCloskey, J. A.; Whitehill, A. B.; Rozenski, J.; Qiu, F.; Crain, P. F. New Techniques for the Rapid Characterization of Oligonucleotides by Mass Spectrometry. Nucleosides Nucleotides 1999, 18, 1549-1553.

12. Ni, J.; Pomerantz, C.; Rozenski, J.; Zhang, Y.; McCloskey, J. A. Interpretation of Oligonucleotide Mass Spectra for Determination of Sequence Using Electrospray Ionization and Tandem Mass Spectrometry. Anal. Chem. 1996, 68, 1989-1999.

13. Hossain, M.; Limbach, P. A. Mass Spectrometry-Based Detection of Transfer RNAs by Their Signature Endonuclease Digestion Products. RNA (A publication of the RNA Society) 2007, 13, 295-303.

14. Durairaj, A.; Limbach. P. A. Matrix-Assisted Laser Desorption/Ionization Mass Spectrometry Screening for Pseudouridine in Mixtures of Small RNAs by Chemical Derivatization, RNase Digestion, and Signature Products. Rapid Commun. Mass Spectrom. 2008, 22, 3727-3734.

15. Cayama, E.; Yepez, A.; Rotondo, F.; Bandeira, E.; Ferreras, A. C.; Triana-Alonso, F. J. New Chromatographic and Biochemical Strategies for Quick Preparative Isolation of tRNA. Nucleic Acids Res. 2000, 28, E64.

16. Loo, J. A.; Quinn, J. P.; Ryu, S. I.; Henry, K. D.; Senko, M. W. McLafferty, F. W. High-Resolution Tandem Mass Spectrometry of Large Biomolecules. Proc. Natl. Acad. Sci. U.S.A. 1992, 89, 286-289.

17. Kelleher, N. L.; Lin, H. Y.; Valaskovic, G. A.; Aaserud, D. J.; Fridriksson, E. K.; McLafferty, F. W. Top-Down Versus Bottom-Up Protein Characterization by Tandem High-Resolution Mass Spectrometry. J. Am. Chem. Soc. 1999, 121, 806-812.

18. Kelleher, N. L. Top-Down Proteomics. Anal. Chem. 2004, 76, 197A-203A.

19. Siuti, N.; Kelleher, N. L. Decoding Protein Modifications Using TopDown Mass Spectrometry. Nature Methods 2007, 4, 817-821.

20. Little, D. P.; Aaserud, D. J.; Valaskovic, G. A.; McLafferty, F. W. Sequence Information from 42-108-mer DNAs (Complete for a 50-mer) by Tandem Mass Spectrometry. J. Am. Chem. Soc. 1996, 118, 9352-9359.

21. Wu, J.; McLuckey, S. A. Gas-Phase Fragmentation of Oligonucleotide Ions. Int. J. Mass Spectrom. 2004, 237, 197-241.

22. Kirpekar, F.; Krogh, T. N. RNA Fragmentation Studied in a MatrixAssisted Laser Desorption/Ionization Tandem Quadrupole/Orthogonal Time-of-Flight Mass Spectrometer. Rapid Commun. Mass Spectrom. 2001, 15, 8-14.

23. Andersen, T. E.; Kirpekar, F.; Haselmann, K. F. RNA Fragmentation in MALDI Mass Spectrometry Studied by H/D-Exchange: Mechanisms of 
General Applicability to Nucleic Acids. J. Am. Soc. Mass Spectrom. 2006, 17, 1353-1368.

24. Schürch, S.; Bernal-Mendez, E.; Leumann, C. J. Electrospray Tandem Mass Spectrometry of Mixed-Sequence RNA/DNA Oligonucleotides. J. Am. Soc. Mass Spectrom. 2002, 13, 936-945.

25. Tromp, J. M.; Schürch, S. Gas-Phase Dissociation of Oligoribonucleotides and Their Analogues Studied by Electrospray Ionization Tandem Mass Spectrometry. J. Am. Soc. Mass Spectrom. 2005, 16, 1262-1268.

26. Huang, T.-Y.; Kharlamova, A.; Liu, J.; McLuckey, S. A. Ion Trap Collision-Induced Dissociation of Multiply Deprotonated RNA: c/yIons Versus (a-B)/w-Ions. J. Am. Soc. Mass Spectrom. 2008, 19, 1832-1840.

27. Huang, T.-Y.; Liu, J.; Liang, X. Hodges, B. D. M.; McLuckey, S. A Collision-Induced Dissociation of Intact Duplex and Single-Stranded siRNA Anions. Anal. Chem. 2008, 80, 8501-8508.

28. Kellersberger, K. A.; Yu, E.; Kruppa, G. H.; Young, M. M.; Fabris, D. Top-Down Characterization of Nucleic Acids Modified by Structural Probes Using High-Resolution Tandem Mass Spectrometry and Automated Data Interpretation. Anal. Chem. 2004, 76, 2438-2445.

29. Griffey, R. H.; Hofstadler, S. A.; Sannes-Lowery, K. A.; Ecker, D. J.; Crooke, S. T. Determinants of Aminoglycoside-Binding Specificity for rRNA by Using Mass Spectrometry. Proc. Natl. Acad. Sci. U.S.A. 1999, 96, 10129-10133.

30. Turner, K. B.; Hagan, N. A.; Kohlway, A. S.; Fabris, D. Mapping Noncovalent Ligand Binding to Stem Loop Domains of the HIV-1 Packaging Signal by Tandem Mass Spectrometry. J. Am. Soc. Mass Spectrom. 2006, 17, 1401-1411.

31. Gabelica, V.; De Pauw, E. Comparison Between Solution-Phase Stability and Gas-Phase Kinetic Stability of Oligodeoxynucleotide Duplexes. J. Mass Spectrom. 2001, 36, 397-402.

32. Gabelica, V.; De Pauw, E. Comparison of the Collision-Induced Dissociation of Duplex DNA at Different Collision Regimes: Evidence for a Multistep Dissociation Mechanism. J. Am. Soc. Mass Spectrom. 2002, 13, 91-98.

33. Xia, Y.; Chrisman, P. A.; Erickson, D. E.; Liu, J.; Liang, X.; Londry, F. A.; Yang, M. J.; McLuckey, S. A. Implementation of Ion/Ion Reactions in a Quadrupole/Time-of-Flight Tandem Mass Spectrometer. Anal. Chem. 2006, 78, 4146-4154.

34. Huber, C. G.; Buchmeiser, M. R. On-Line Cation Exchange for Suppression of Adduct Formation in Negative-Ion Electrospray Mass Spectrometry of Nucleic Acids. Anal. Chem. 1998, 70, 5288-5295.
35. Greig, M.; Griffey, R. H. Utility of Organic-Bases for Improved Electrospray Mass-Spectrometry of Oligonucleotides. Rapid Commun. Mass Spectrom. 1995, 9, 97-102.

36. Liang, X.; Xia, Y.; McLuckey, S. A. Alternately Pulsed Nanoelectrospray Ionization/Atmospheric Pressure Chemical Ionization for Ion/Ion Reactions in an Electrodynamic Ion Trap. Anal. Chem. 2006, 78, 3208-3212.

37. Sprinzl, M.; Vassilenko, K. S. Compilation of tRNA Sequences and Sequences of tRNA Genes. Nucleic Acids Res. 2005, 33(Database issue),D139-140.

38. Rozenski, J. Nucleic Acids Masspec Toolbox (Mongo Oligo Mass Calculator v2.06). http://medlib.med.utah.edu/masspec.

39. Rajbhandary, U. L.; Chang, S. H.; Stuart, A.; Faulkner, R. D.; Hoskinson, R. M.; Khorana, H. G. Studies on Polynucleotides, LXVIII. The Primary Structure of Yeast Phenylalanine Transfer RNA. Proc. Natl. Acad. Sci. U.S.A. 1967, 57, 751-758.

40. Wimmer, E.; Maxwell, I. H.; Tener, G. M. A Simple Method for Isolating Highly Purified Yeast Phenylalanine Transfer Ribonucleic Acid. Biochemistry 1968, 7, 2623-2628.

41. Wu, J.; McLuckey, S. A. Ion/Ion Reactions of Multiply Charged Nucleic Acid Anions: Electron Transfer, Proton Transfer, and Ion Attachment. Int. J. Mass Spectrom. 2003, 228, 577-597.

42. McLuckey, S. A.; Reid, G. E.; Wells, J. M. Ion Parking During Ion/Ion Reactions in Electrodynamic Ion Traps. Anal. Chem. 2002, 74, 336-346.

43. Patteson, K. G.; Rodicio, L. P.; Limbach, P. A. Identification of the Mass-Silent Post-Transcriptionally Modified Nucleoside Pseudouridine in RNA Base Matrix-Assisted Laser Desorption/Ionization Mass Spectrometry. Nucleic Acids Res. 2001, 29, e49.

44. Han, X. M.; Jin, M.; Breuker, K.; McLafferty, F. W. Extending Top-Down Mass Spectrometry to Proteins with Masses Greater than 200 Kilodaltons. Science 2006, 314, 109-112.

45. Mattick, J. S. Noncoding RNAs: The Architects of Eukaryotic Complexity. EMBO Rep. 2001, 2, 986-991.

46. Mattick, J. S. The Functional Genomics of Noncoding RNA. Science 2005, 309, 1527-1528.

47. Miyauchi, K.; Ohara, T.; Suzuki, T. Automated Parallel Isolation of Multiple Species of Noncoding RNAs by the Reciprocal Circulating Chromatography method. Nucleic Acids Res. 2007, 35, e24.

48. Yu, B.; Yang, Z.; Li, J.; Minakhina, S.; Yang, M.; Padgett, R. W.; Steward, R.; Chen, X. Methylation as a Crucial Step in Plant microRNA Biogenesis. Science 2005, 307, 932-935. 